\title{
FAR-IR SPECTROPHOTOMETRY OF HH FLOWS WITH THE ISO LONG-WAVELENGTH SPECTROMETER
}

\author{
R. LISEAU \\ Stockholm Observatory \\ S-133 36 Saltsjöbaden, Sweden \\ e-mail: rene@astro.su.se \\ T. GIANNINI, B. NISINI, P. SARACENO AND L. SPINOGLIO \\ CNR-Istituto di Fisica dello Spazio Interplanetario \\ I-000 44 Frascati, Italy \\ B. LARSSON \\ Stockholm Observatory \\ S-133 36 Saltsjöbaden, Sweden \\ D. LORENZETTI \\ Osservatorio Astronomico di Roma \\ I-000 40 Monte Porzio, Italy \\ AND \\ E. TOMMASI \\ Lws Instrument Dedicated Team \\ Iso Science Operations Centre \\ Villafranca de Castillo, Spain
}

\begin{abstract}
Full Iso-Lws spectral scans between about 45 to $190 \mu \mathrm{m}$ of 17 individual $\mathrm{HH}$ objects in 7 star forming regions have revealed essentially only [O I] $63 \mu \mathrm{m}$ line emission, implying that the FIR cooling of these objects is totally dominated by this line alone. In this case, J-shock models can be used to determine the mass loss rates of the $\mathrm{HH}$ exciting sources. These mass loss rates are in reasonably good agreement with those estimated for the accompanying $\mathrm{CO}$ flows, providing first observational evidence that $\mathrm{HH}$ and molecular flows are driven by the same agent. The $L_{\text {mech }}-L_{\text {bol }}$ relation, based on our results with the LWS, implies that young stellar objects of lower mass are loosing mass at relatively higher rates than their more massive counterparts.
\end{abstract}




\section{Introduction}

Herbig-Haro objects (HH) have commonly been identified on the basis of observations in the visual spectral region. As such, the known objects are, when viewed against dark clouds, surface phenomena in regions of stellar formation. Recent advances in detector array development have opened the near infrared (NIR) for the observational study of HH flows and a growing body of $2 \mu \mathrm{m}$-data is now becoming available also for cloud embedded flows (cf. article by J. Eislöffel). At NIR-wavelengths, extinction of radiation by dust can still present a problem, particularly in regions of high column density $\left(A_{\mathrm{K}} \sim 0.1 A_{\mathrm{V}}\right)$, and $\mathrm{HH}$ flows deeply embedded or on the far side of the clouds might not be readily accessible to NIR observations. On the other hand, at far infrared (FIR) wavelengths, extinction is generally negligible ( $A_{100 \mu \mathrm{m}} \lesssim 10^{-3} A_{\mathrm{V}}$ ), except perhaps on the very smallest spatial (subarcsec) scales.

First FIR spectroscopic observations of $\mathrm{HH}$ flows have been presented by Cohen et al. (1988). These data were obtained from airplane altitude (KAO) and were restricted, therefore, to the few existing spectral windows of diminished telluric opacity. The space borne Lws, in contrast, is free of atmospheric limitations and has observed, for the first time, fully covering FIR-spectra of HH flows.

\subsection{PRIMARY OBJECTIVES OF THE LWS-HH PROGRAMME}

The Lws (Long-Wavelength Spectrometer: Clegg et al. 1996, Swinyard et al. 1996) aboard Iso (Infrared Space Observatory: Kessler et al. 1996) operates in the wavelength region $\sim 40-200 \mu \mathrm{m}$ and FIR-spectrophotometry of unextinguished $\mathrm{HH}$ flows offered thus the opportunity to address:

- the spatial distribution (extent) and morphology (degree of symmetry) of HH flows;

- the appearance of FIR-spectra and the importance of FIR line cooling (atomic and molecular) for the overall energy balance of $\mathrm{HH}$ flows;

- the nature of the HH-excitation in molecular clouds, i.e. to what degree the shocks are predominantly J- or C-type;

- the physics of mass loss from young stellar objects and the interrelation of molecular ('CO ouflows') and $\mathrm{HH}$ flows.

In addition, since $\mathrm{HH}$ objects were expected to emit little, if any, continuous radiation at long wavelengths, Lws observations should also be suitable to obtain the FIR energy distributions of the associated clouds at high spectral resolution. This should permit the study of the dust opacity in dark, molecular clouds and the search for solid state spectral features in a previously unexplored wavelength regime. 


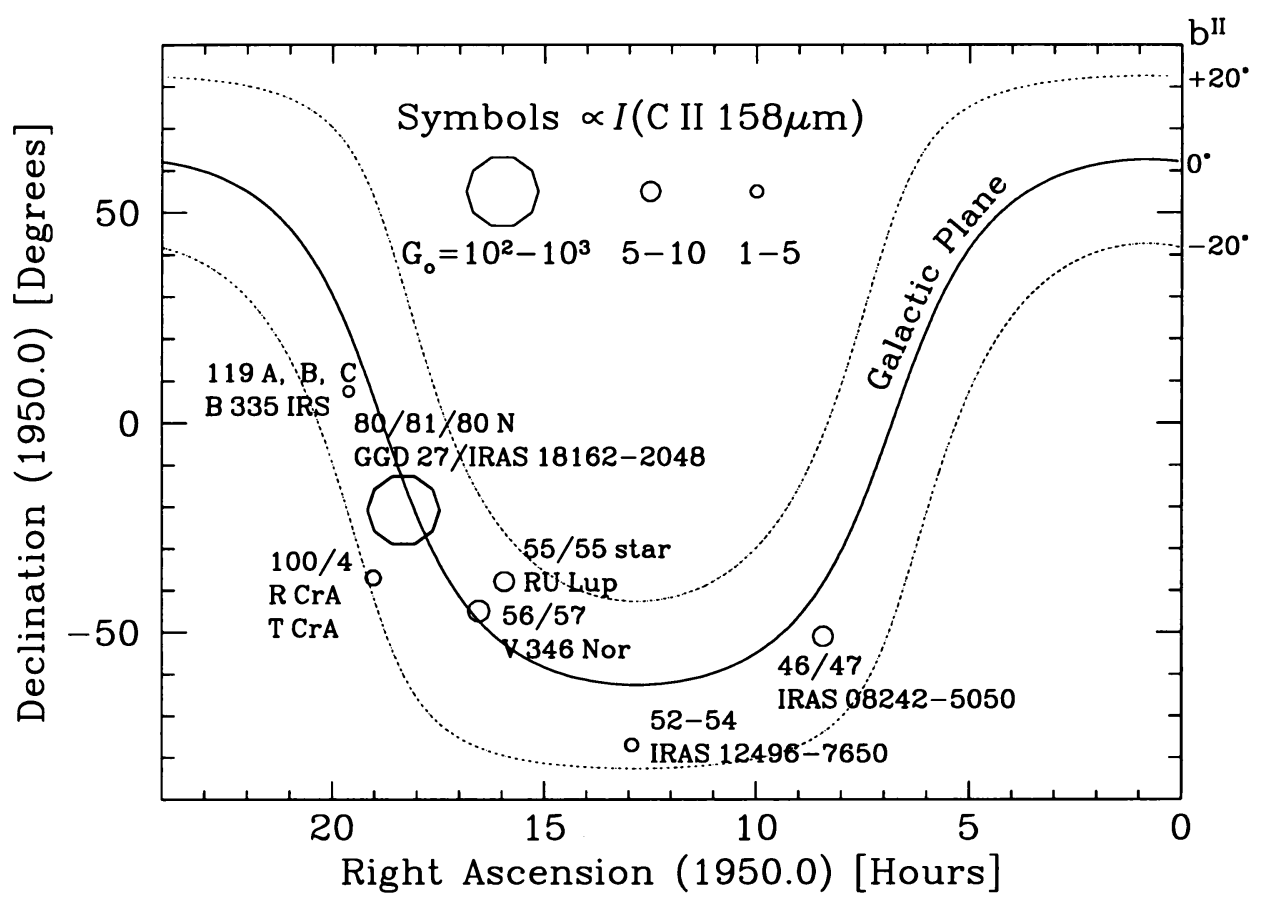

Figure 1. The sky distribution, in equatorial coordinates, of $17 \mathrm{HH}$ flows in 7 star forming regions observed with the Lws is shown together with the driving stellar sources. Symbol sizes correspond roughly to the value of the interstellar radiation field, expressed in terms of $G_{0}$ (see text). For convenience, the run of the galactic plane and of the galactic latitudes $\pm 20^{\circ}$ are also shown

In this contribution, we concentrate mainly on the aspects of shock-type and mass loss during the early phases of stellar evolution.

\section{HH observations with the LWS}

The data presented in this contribution have been obtained during Guaranteed Time observations of the Lws-team. In 7 different regions of star formation, the number of individual $\mathrm{HH}$ objects observed was 17 and their distribution in the sky is shown in Fig. 1. The lack of objects in the constellations of, e.g., Perseus, Taurus-Auriga and Orion reflects the visibility restrictions of IsO. HH flows in these regions are expected to be observed during the extended Iso-mission in 1997/98.

Full Lws-grating scans $(43-193 \mu \mathrm{m})$ have been obtained for all objects, at the spectral resolution $R_{\lambda} \sim 150-300$. The observations with the $80^{\prime \prime}$ circular beam were made both in pointed and in mapping mode, i.e. point- 


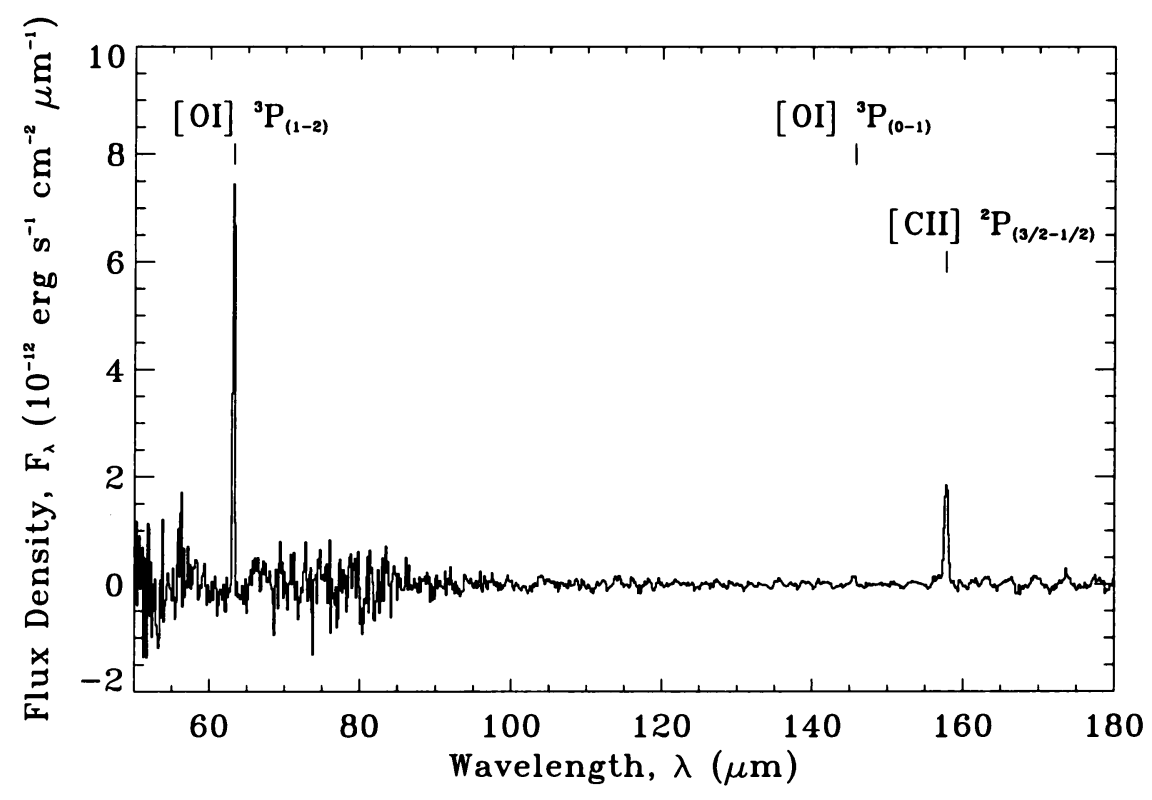

Figure 2. Typical FIR line spectrum of $\mathrm{HH}$ objects

ing the telescope towards optically identified $\mathrm{HH}$ positions as well as obtaining unbiased raster scan maps of the flows. The absolute flux calibration is believed to be better than about $30 \%$. Within integration times of about 700 to $4500 \mathrm{~s}$ per position a continuum sensitivity $(1 \sigma)$ of $\sim 1 \mathrm{Jy}$ at 60 and $100 \mu \mathrm{m}$ was achieved, comparable to that of IRAS at much lower spectral resolution. The sensitivity $(1 \sigma)$ to spectral line flux was about $210^{-13} \mathrm{erg} \mathrm{cm}^{-2} \mathrm{~s}^{-1}$ at $60 \mu \mathrm{m}$ and $310^{-14} \mathrm{erg} \mathrm{cm}^{-2} \mathrm{~s}^{-1}$ at $160 \mu \mathrm{m}$ respectively.

\section{Results and discussion}

\subsection{EXTENDED [C II] $158 \mu \mathrm{m}$ EMISSION AND MOLECULAR CLOUD PDR}

First results have been published by Liseau et al. (1996) and by Nisini et al. (1996). These regarded our observations of HH 52-54 and revealed the remarkably rich line spectrum of $\mathrm{HH} 54$, displaying, in addition to atomic fine structure lines, rotational molecular lines of $\mathrm{CO}, \mathrm{OH}$ and $\mathrm{H}_{2} \mathrm{O}$. These latter lines were not seen towards either $\mathrm{HH} 52, \mathrm{HH} 53$ nor in the mapped part of the molecular outflow and it was reasonable to assume that the nondetections were due to insufficient signal-to-noise in these somewhat weaker 
spectra. The Lws observations of the significantly increased sample of $\mathrm{HH}$ flows presented here show, however, that molecular line emission is rarely present in the far infrared spectra of these objects. The median spectrum of the flows (Fig. 2) emphasizes this point: the only lines present at any appreciable strength are due to the fine structure transitions [O I] $63 \mu \mathrm{m}$ and $[\mathrm{C}$ II] $158 \mu \mathrm{m}$.

From the mapping observations it became evident that the [C II] $158 \mu \mathrm{m}$ emission is spatially extended and relatively uniform in intensity, contrary to what is observed for [O I] $63 \mu \mathrm{m}$ being localized and of variable strength.

The relative constancy of the extended [C II] $158 \mu \mathrm{m}$ emission leads us to suspect that this line is not dominated by the HH shocks, but rather originates in the PDR (Photon Dominated Region) of the molecular host clouds. This suspicion is supported by the theoretical predictions for [O I] $63 \mu \mathrm{m}$ and [C II] $158 \mu \mathrm{m}$ of PDR models (Wolfire et al 1990; Hollenbach et al. 1991) and those of J-shock models (Hollenbach \& McKee 1989). As long as the interstellar UV-field, expressed in terms of the local value of the solar neighbourhood, $G_{0}$, does not greatly exceed $10^{3}$, the intensity ratio [C II] $158 \mu \mathrm{m} /[\mathrm{O} \mathrm{I}] 63 \mu \mathrm{m} \gg 1$ in a low density PDR, whereas this ratio is inverted behind fast dissociative J-shocks. From the measured [C II] $158 \mu \mathrm{m}$ intensities towards the $\mathrm{HH}$ flows and the PDR models, we have estimated the values of $G_{0}$ for the different environs. With the exception of $\mathrm{HH} 80-81$, these values are generally in the range 1-10 (cf. Fig. 1).

\section{2. [O I] $63 \mu \mathrm{m}$ LINE LUMINOSITY AND STELLAR MASS LOSS RATE}

The localized character of the [O I] $63 \mu \mathrm{m}$ emission certainly associates it with the $\mathrm{HH}$ objects. As evidenced in Fig. 2, the level of molecular line emission (in particularly $\mathrm{CO}$ and $\mathrm{H}_{2} \mathrm{O}$ ) is very low compared to that in [O I] $63 \mu \mathrm{m}$. From comparison with the theoretical models of C-shocks by Draine et al. (1983) we infer that HH shocks appear generally not to be dominated by the magnetically supported, non-dissociative C-type. Rather, our Lws observations demonstrate that the FIR cooling of these flows is completely dominated by [O I] $63 \mu \mathrm{m}$ emission. For the $\mathrm{HH}$ excitation, our data are in favour, therefore, of dissociative J-shock models (Hollenbach \& McKee 1989).

It was realized by Hollenbach (1985; see also: Hollenbach \& McKee 1989) that, in certain conditions, the [O I] $63 \mu \mathrm{m}$ line luminosity is a direct measure of the rate at which the central stellar source looses mass. Specifically, for a dissociative 'wind shock' with particle flux, $\Phi_{\mathrm{H}}$, expressed in terms of the pre-shock particle density $n_{0}$ and the shock velocity $v_{\mathrm{s}}$, such that

$$
\left[\frac{\Phi_{\mathrm{H}}}{\mathrm{cm}^{-2} \mathrm{~s}^{-1}}\right] \lesssim 1.0\left[\frac{n_{0}}{10^{5} \mathrm{~cm}^{-3}}\right]\left[\frac{v_{\mathrm{s}}}{100 \mathrm{~km} \mathrm{~s}^{-1}}\right]
$$




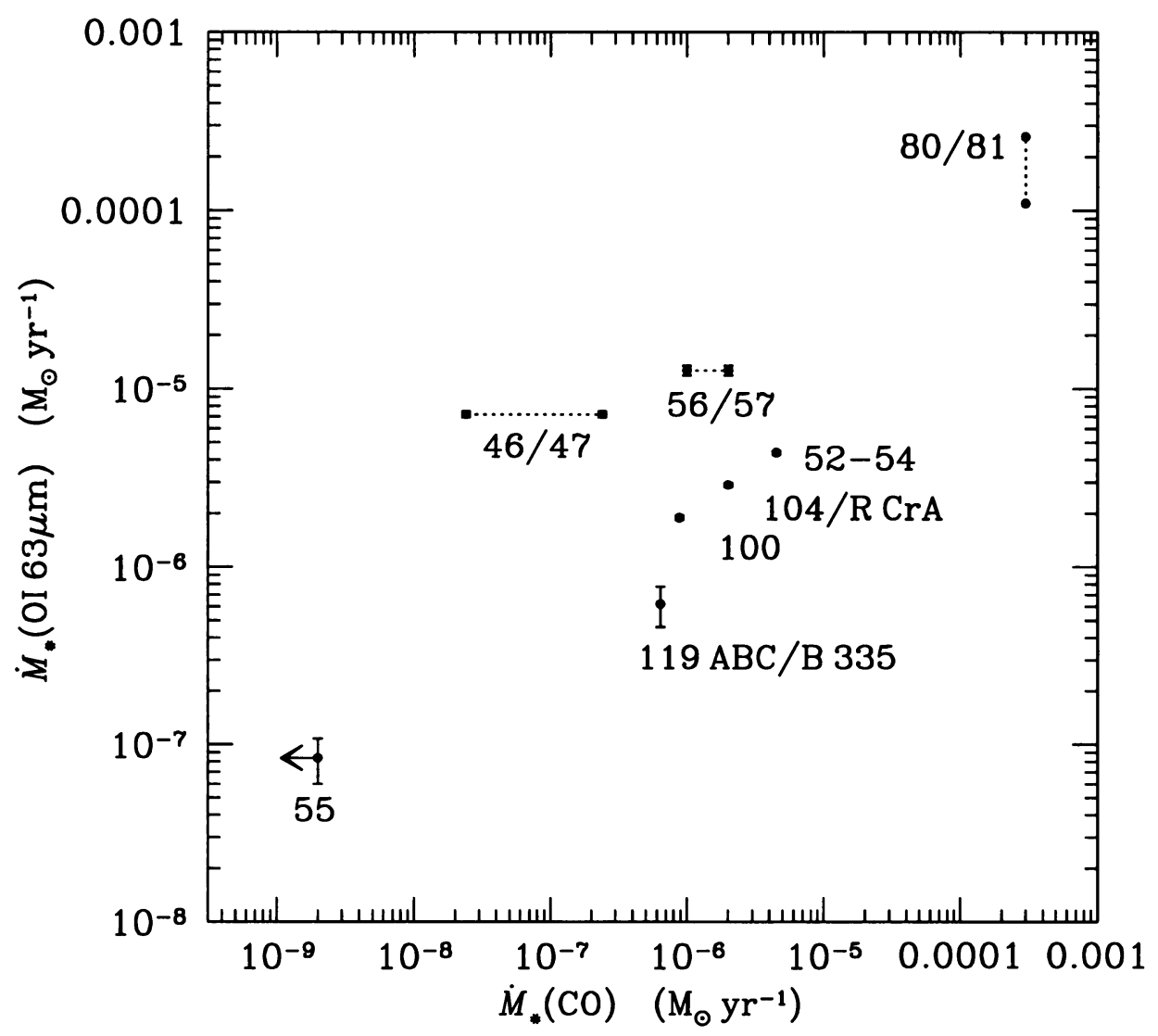

Figure 3. Stellar mass loss rates, based on observations of [O I] $63 \mu \mathrm{m}$ and on $\mathrm{CO}$ observations taken from the literature, for the HH flows observed with the Lws

the $[\mathrm{O}$ I] $63 \mu \mathrm{m}$ luminosity is directly proportional to the stellar mass loss rate, viz.

$$
\dot{M}_{*}=10^{-6}\left[\frac{L(\mathrm{OI} 63 \mu \mathrm{m})}{10^{-2} \mathrm{~L}_{\odot}}\right] \quad\left(\mathrm{M}_{\odot} \mathrm{yr}^{-1}\right) .
$$

Similarly, from spatial maps of the wing intensity of mm-wave CO lines, estimates of $\dot{M}_{*}$ can be deduced, commonly by assuming (for low luminosity sources) momentum to be conserved, viz. $\dot{P}=$ constant, so that

$$
\dot{M}_{*}=10^{-6}\left[\frac{M}{0.1 \mathrm{M}_{\odot}}\right]\left[\frac{\Delta v}{10 \mathrm{~km} \mathrm{~s}^{-1}}\right]\left[\frac{\Delta t}{10^{4} \mathrm{yr}}\right]^{-1}\left[\frac{v_{\infty}}{100 \mathrm{~km} \mathrm{~s}^{-1}}\right]^{-1}\left(\mathrm{M}_{\odot} \mathrm{yr}^{-1}\right)
$$




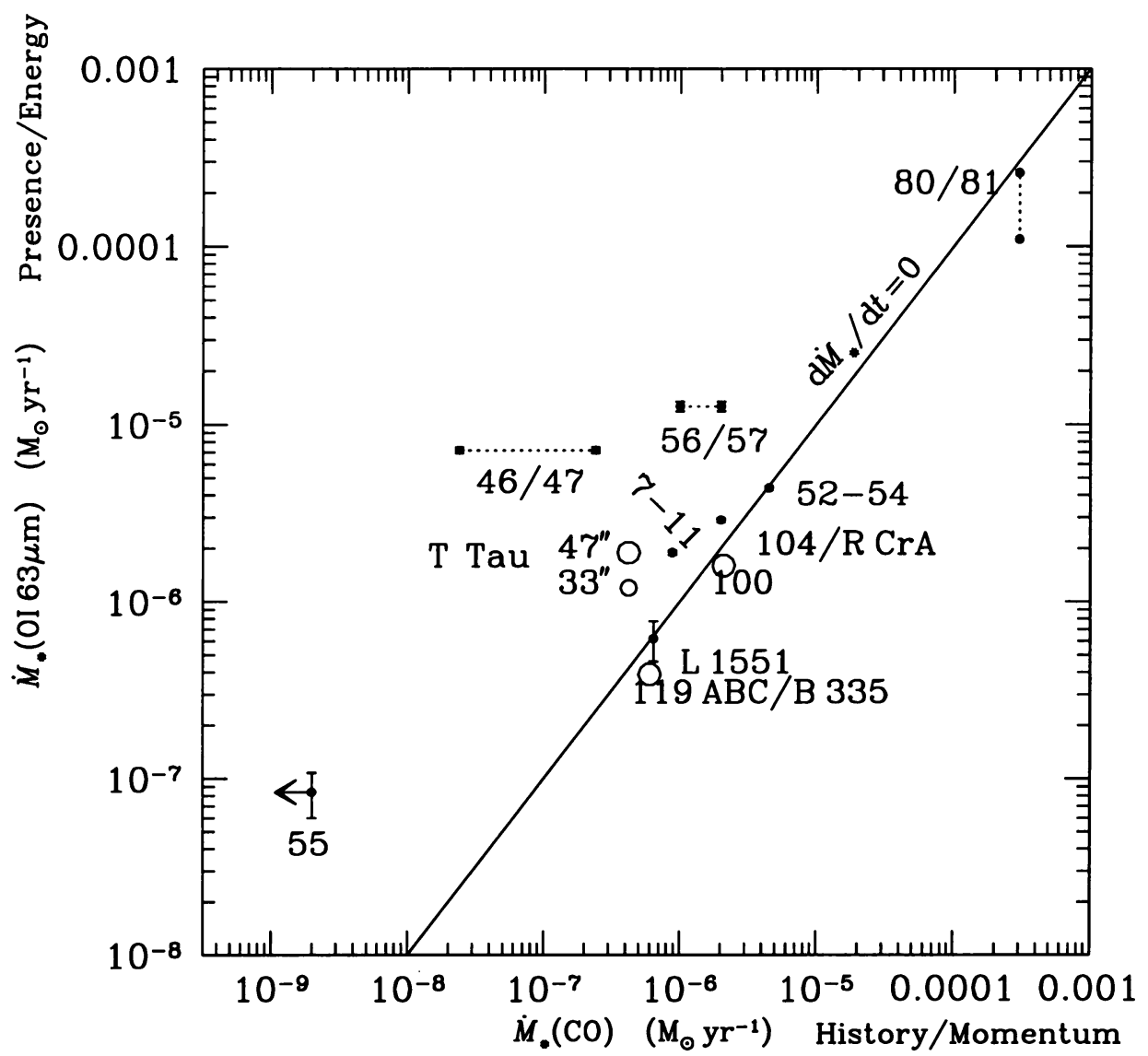

Figure 4. Same as Fig. 3 but data for HH 7-11, L 1551 IRS 5 and T Tau (Cohen et al. 1988) are also shown (open symbols). In addition, a line of unit slope is shown for reference

where $M$ and $\Delta v$ are the mass and the velocity of the molecular gas, respectively, $\Delta t$ is an estimate of the CO flow time scale and $v_{\infty}$ is the terminal velocity of the stellar wind which has accelerated the molecular gas. Neglecting contributions from the CO line core, not accounting for opacity and/or projection effects will imply uncertainties of the mass loss rates as large as more than one order of magnitude (e.g. Cabrit \& Bertout 1992). Further, what regards distance uncertainties, the dependencies for these two methods are different, viz. $\dot{M}_{*}([\mathrm{OI}] 63 \mu \mathrm{m}) \propto D^{2}$, whereas $\dot{M}_{*}(\mathrm{CO}) \propto D$. 


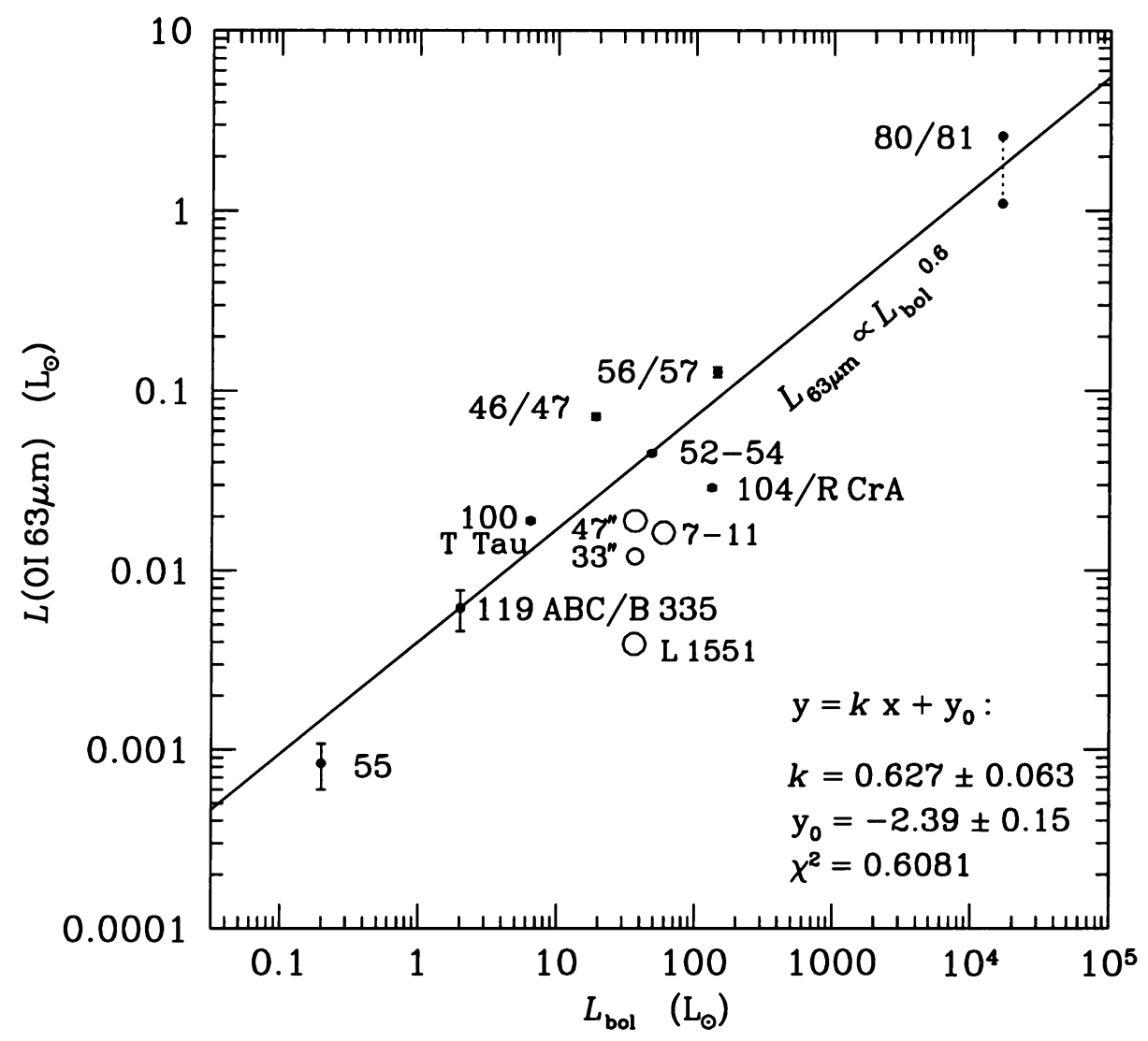

Figure 5. Luminosity in [O I] $63 \mu \mathrm{m}$ versus stellar $L_{\mathrm{bol}}$. The former is directly proportional to the stellar mass loss rate, $M$. Filled points are Lws data, whereas open symbols refer to data by Cohen et al., shown for comparison. The least squares fit, shown by the straight line, refers to Lws data only

Given these sources of large uncertainty we would expect considerable scatter in a $\dot{M}_{*}([\mathrm{O} \mathrm{I}] 63 \mu \mathrm{m})-\dot{M}_{*}(\mathrm{CO})$ plot. However, the contrary is revealed by Fig. 3 showing these quantities to correlate. This trend is clearly supported by data from Cohen et al. (1988), which are shown superposed in Fig. 4.

Also shown in Fig. 4, for reference, is a straight line of unit slope, designating the locus of complete congruence between $\dot{M}_{*}([\mathrm{O} \mathrm{I}] 63 \mu \mathrm{m})$ and $M_{*}(\mathrm{CO})$ (note that this is not a fit to the data points).

These two determinations of $\dot{M}_{*}$ refer to two different aspects of the 
mass loss, though. In the [O I] $63 \mu \mathrm{m}$ emission, the instantaneous energy dissipation is observed, whereas the $\mathrm{CO}$ flows measure the momentum transfer over time scales, $\Delta t$, much longer than the cooling time of [O I] $63 \mu \mathrm{m}$. One interpretation of the straight line would be, then, that of temporal constancy of the mass loss from the central stellar source. According to the figure, for most sources $\dot{M}_{*}$ seems not to vary much. Interestingly, however,

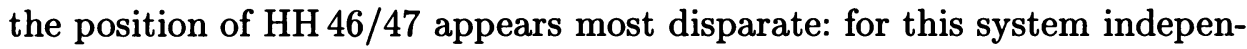
dent evidence for time variable flows does exist (e.g. Hartigan et al. 1993).

\section{3. [O I] $63 \mu \mathrm{m}$ LINE AND STELLAR BOLOMETRIC LUMINOSITY}

One 'prediction' of the correlation of Figs. 3 and 4 is that there should exist a Lada-relation for outflows (Lada 1985), i.e. for the mechanical luminosity (the [O I] $63 \mu \mathrm{m}$ luminosity) and the bolometric luminosity of the mass loosing stellar source. Such dependence is indeed implied by the data displayed in Fig. 5. Formally, our Lws data yield (with luminosities in $\mathrm{L}_{\odot}$ )

$$
L_{63 \mu \mathrm{m}} \sim 0.004 L_{\mathrm{bol}}^{0.6} \text {. }
$$

This power law exponent, 0.6, is identical to that found by Edwards et al. (1993) for stellar jets, and similar to that (0.8) determined by Cabrit \& Bertout (1992) for CO outflows, but contrasting to earlier findings, according to which exponents $\gtrsim 1$ have commonly been inferred (see, e.g., review by Bally \& Lane 1991). These works were generally biased towards high luminosity objects. Consequently, the more recent findings suggest that low luminosity (low mass) young stellar objects are relatively more efficiently 'generating' mass loss than their high mass counterparts. However, whether this could mean that the mass loss processes (the 'drivings') are different in this stellar mass dichotomy, is not possible to tell on the basis of the presented observational evidence.

\section{Conclusions}

Iso-Lws observations of $17 \mathrm{HH}$ Objects in 7 regions of stellar formation lead us to conclude:

- The FIR spectra of HH flows show a general lack of prominent molecular line emission, indicating that magnetically supported shocks do not play a dominant rôle in $\mathrm{HH}$ excitation.

- The uniformly extended [C II] $158 \mu \mathrm{m}$ line emission from (low luminosity) $\mathrm{HH}$ regions can generally be attributed to weak PDRs at the cloud surface $\left(G_{0} \sim 1-10\right)$.

- In mapped flows, the [O I] $63 \mu \mathrm{m}$ line displays strong spatial variations, apparently related with optically identified $\mathrm{HH}$ emission. 
- Comparison of stellar mass loss rates estimated using the [O I] luminosity with those determined from mm-wave $\mathrm{CO}$ observations results in remarkable agreement, providing empirical proof that $\mathrm{HH}$ flows and molecular flows ('CO outflows') are physically associated.

- These data provide also a demonstration of the potential of the [O I] $63 \mu \mathrm{m}$ line as a mass loss diagnostic.

- The [O I] luminosity, $L([\mathrm{O} \mathrm{I}] 63 \mu \mathrm{m})$, being a measure of the stellar mass loss rate, correlates with the bolometric luminosity, $L_{\mathrm{bol}}$, of the central driving source.

- From this relation it is inferred that low mass young stellar objects are more efficient 'mass loosers' than are young stars of high mass.

\section{References}

Bally J., Lane A.P., 1991, in: Lada C.J. \& Kylafis N.D. (eds.) The physics of star formation and early stellar evolution, Kluwer, p. 471

Cabrit S., Bertout C., 1992, A\&A 261, 274

Clegg P.E. et al., 1996, A\&A 315, L 38

Cohen M., Hollenbach D.J., Haas M.R., Erickson E.F., 1988, ApJ 329, 863

Draine B.T., Roberge W.G., Dalgarno A., 1983, ApJ 264, 485

Edwards S., Ray T., Mundt R., 1993, in: E.H. Levy \& Lunine J.I. (eds.), Protostars and Planets III, U.Arizona, p. 567

Hartigan P., Morse J.A., Heathcote S., Gerald C., 1993, ApJ 414, L 121

Hollenbach D.J., 1985, Icarus 61, 36

Hollenbach D., McKee C.F., 1989, ApJ 342, 306

Hollenbach D.J., Takahashi T., Tielens A.G.G.M., 1991, ApJ 377, 192

Kessler M. et al., 1996, A\&A 315, L 27

Lada C.J., 1985, ARA\&A 23, 267

Liseau R. et al., 1996, A\&A 315, L 181

Nisini B. et al., 1996, A\&A 315, L 321

Swinyard B.M. et al., 1996, A\&A 315, L 43

Wolfire M.G., Tielens A.G.G.M., Hollenbach D., 1990, ApJ 358, 116 